\title{
Transcription regulates telomere dynamics in human cancer cells
}

\author{
RAJIKA ARORA, CATHERINE M. BRUN, and CLAUS M. AZZALIN ${ }^{\mathbf{1}}$ \\ Institute of Biochemistry, Eidgenössische Technische Hochschule Zürich (ETHZ), CH-8093 Zürich, Switzerland
}

\begin{abstract}
Telomeres are nucleoprotein structures capping the physical ends of linear eukaryotic chromosomes. Although largely heterochromatic, telomeres are transcribed into telomeric repeat-containing RNA (TERRA) molecules by RNA polymerase II. The functions associated with telomere transcription and TERRA remain ill defined. Here we show that the transcriptional activity of human telomeres directly regulates their movement during interphase. We find that chemical inhibition of global transcription dampens telomere motion, while global stimulation promotes it. Likewise, when DNA methyltransferase enzymes are deleted to augment telomere transcription, we observe increased telomere movement. Finally, using a cell line engineered with a unique transcriptionally inducible telomere, we show that transcription of one specific telomere stimulates only its own dynamics without overtly affecting its stability or its length. We reveal a new and unforeseen function for telomere transcription as a regulator of telomere motion, and speculate on the intriguing possibility that transcription-dependent telomere motion sustains the maintenance of functional and dysfunctional telomeres.
\end{abstract}

Keywords: telomeres; transcription; TERRA; chromatin dynamics

\section{INTRODUCTION}

Telomeres, the heterochromatic structures located at the end of linear eukaryotic chromosomes, are transcribed by DNA-dependent RNA polymerase II (RNAPII) into telomeric repeat-containing RNA (TERRA) molecules (Solovei et al. 1994; Azzalin et al. 2007; Luke et al. 2008; Schoeftner and Blasco 2008; Arora et al. 2010; Vrbsky et al. 2010). In human cells, the large majority of TERRA is transcribed from $\mathrm{CpG}$ dinucleotide-rich islands located on approximately half of the subtelomeres (Nergadze et al. 2009). During the past few years, different functions have been proposed for TERRA. Biochemical in vitro assays suggested that TERRA might regulate telomere replication through inhibition of telomerase activity at chromosome ends, as well as by coordinating the cell cycle-dependent exchange of the two single-stranded DNA-binding proteins, RPA and POT1, at telomeres (Schoeftner and Blasco 2008; Redon et al. 2010; Flynn et al. 2011). In addition, short interference (si) RNA-mediated down-regulation of TERRA cellular levels led to decreased density of heterochromatin

\footnotetext{
${ }^{1}$ Corresponding author.

E-mail claus.azzalin@bc.biol.ethz.ch.

Article published online ahead of print. Article and publication date are at http://www.rnajournal.org/cgi/doi/10.1261/rna.029587.111.
}

marks at telomeres and to telomeric aberrations in human cancer cells. Hence, TERRA might promote telomeric heterochromatin deposition and telomere stability (Deng et al. 2009).

Mammalian telomeres are not immobile, but, as other chromatin loci, move within the nuclear territory (Molenaar et al. 2003; Dimitrova et al. 2008; Wang et al. 2008; Jegou et al. 2009; Stixova et al. 2011). The motility of individual telomeres within the same cell is largely heterogeneous and inversely correlates with their length. While long telomeres possess slow motion similar to the constrained movement of centromeric chromatin, short telomeres move faster and forced telomere elongation induced a sharp decrease in telomere dynamics (Molenaar et al. 2003; Wang et al. 2008). In contrast, telomere uncapping achieved through gene deletion of the telomeric factor TRF2 or overexpression of a mutant-template telomerase RNA increased telomere motility, suggesting a functional link between telomere integrity and telomere movements (Dimitrova et al. 2008; Wang et al. 2008).

Studies on fixed mammalian cells indicate that the position of chromosomes within the nucleus correlates with their transcriptional activity. Gene-poor chromosomes preferentially localize in proximity of the nuclear periphery, while gene-rich chromosomes are closer to the nucleus center (Boyle et al. 2001). A similar correlation between 
nuclear organization and transcription is also known to exist at the level of individual genes (Zhao et al. 2009; Rajapakse and Groudine 2011). Live imaging of mammalian cells shows that the movement of heterochromatic domains is more constrained than the one of euchromatin, and this difference in motility is thought to arise from different transcriptional activities associated with heterochromatin and euchromatin (Hübner and Spector 2010). Consistent with this assumption, chromatin localized near the nuclear periphery tends to move less than chromatin positioned more internally in the nucleus (Chubb et al. 2002). In addition, a transcriptionally silent transgene integrated into hamster chromosomes moved with highly constrained and nondirected dynamics in proximity of the nuclear periphery. Transcription induction of the same transgene led to its fast repositioning from the nuclear periphery to the interior through long-range, directed movements (Chuang et al. 2006). Altogether, these observations prompted us to test whether transcription of a telomere regulates its motion.

\section{RESULTS AND DISCUSSION}

\section{Transcription influences telomere dynamics in human cancer cells}

We infected HeLa cells with retroviruses expressing the telomeric factor TRF1 fused in-frame with green fluorescence protein (GFP-TRF1). GFP-TRF1 extensively colocalized with the endogenous telomeric protein hRap1 (Fig. 1A). GFP-TRF1 was $\sim 11$-fold overexpressed above endogenous TRF1 (Supplemental Fig. S1A) and, consistent with TRF1 negatively regulating telomere length (van Steensel and de Lange 1997), infected cells contained telomeres more heterogeneous in length than uninfected cell controls (Supplemental Fig. S1B). We then treated GFP-TRF1 cells with the global transcription inhibitor Actinomycin D (ActD) for $30 \mathrm{~min}$. The short time of treatment was chosen to avoid secondary effects stemming from impaired transcription of mRNAs coding for factors influencing
A

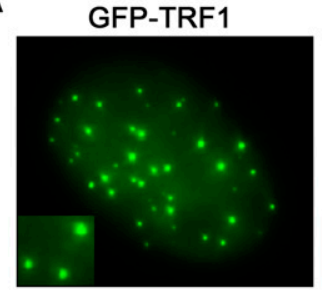

C

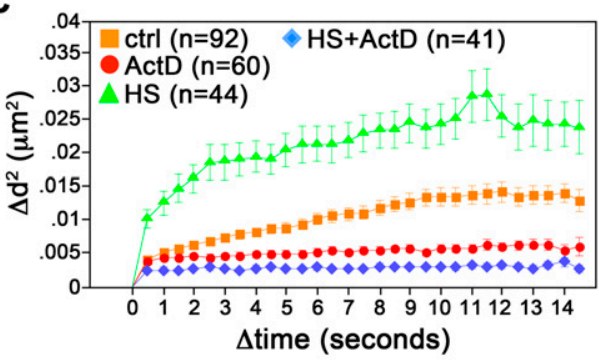

E

\begin{tabular}{|lcc|}
\hline TRT & $\begin{array}{c}\text { ROC }(\mu \mathrm{m}) \\
\Delta \mathrm{t}=7 \mathrm{sec}\end{array}$ & $\begin{array}{c}\mathrm{SS}(\mu \mathrm{m}) \\
\Delta \mathrm{t}=1 \mathrm{sec}\end{array}$ \\
\hline ctrl & 0.10 & 0.056 \\
ActD & 0.07 & 0.050 \\
HS & 0.15 & 0.084 \\
HS+ActD & 0.054 & 0.034 \\
\hline
\end{tabular}

hRap1

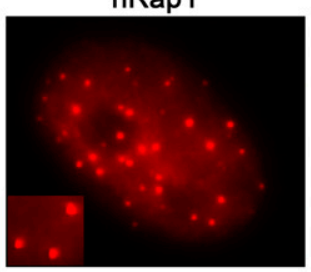

D
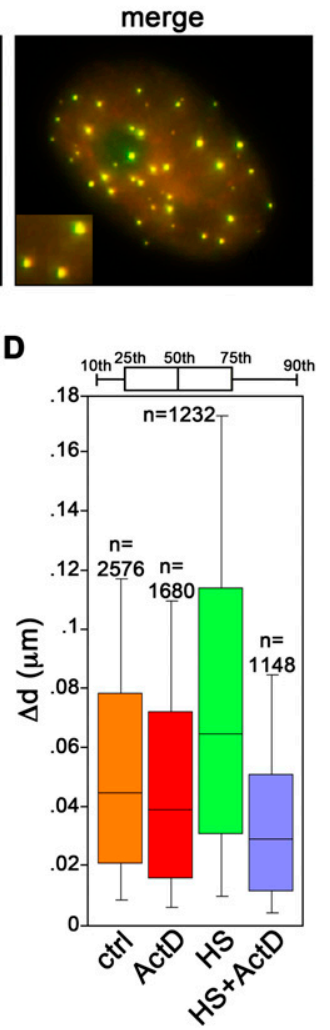

B

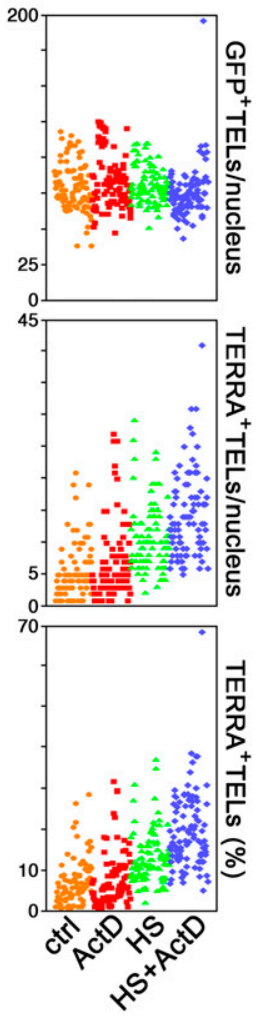

FIGURE 1. Telomere movement correlates with the transcriptional state of the cell. (A) HeLa cells expressing GFP-TRF1 (green) were immunostained for the telomeric factor hRap1 (red). Enlarged regions are shown at the bottom left corners. (B) TERRA RNA FISH analysis of HeLa cells expressing GFP-TRF1. (Top) Total number of GFP-TRF1 foci (GFP ${ }^{+}$TELs) per nucleus; (middle) total number of TERRA-positive

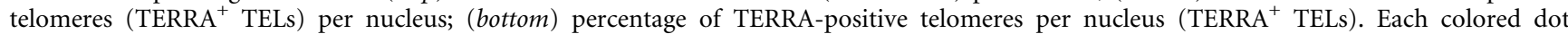
represents data from one nucleus. A total of $\sim 100$ nuclei from two independent experiments were analyzed per treatment. ( $C-E)$ HeLa GFP-TRF1 cells were treated with Actinomycin D (ActD) for 30 min or heat shock (HS) for 60 min, or heat shocked for 60 min and treated with ActD for the last $30 \mathrm{~min}(\mathrm{HS}+\mathrm{ActD})$. Images were taken at a single focal plane every $0.5 \mathrm{sec}$ over $15 \mathrm{sec} .(C)$ Average mean square change in relative distance between two neighboring telomeres. The number of analyzed telomere pairs is indicated (n). (D) Step size (SS) distribution for $\Delta \mathrm{t}$ of 1 sec. The number of data points is indicated (n). (E) Values for radius of confinement (ROC) and SS for the indicated treatments (TRT). All $P$-values are $<0.0001$, and therefore are not indicated. 
chromatin motion or telomere stability. Real time quantitative reverse-transcription polymerase chain reaction (qRTPCR) experiments revealed no significant change in the steady-state levels of TERRA molecules transcribed from $10 \mathrm{q}, 15 \mathrm{q}$, and $\mathrm{Xp} / \mathrm{Yp}$ chromosome ends in ActD-treated cells (Supplemental Fig. S1C). This is in agreement with the previously reported TERRA half-life of $\sim 3 \mathrm{~h}$ (Azzalin et al. 2007). On the contrary, the short-lived c-myc mRNA decreased approximately fivefold (Supplemental Fig. S1C), confirming the functionality of the ActD treatment. We also performed RNA fluorescence in situ hybridization (RNA-FISH) experiments to visualize TERRA in GFP-TRF1 expressing cells. ActD treatment did not substantially affect the average number of GFP-TRF1 foci ( 80 GFP-TRF1 foci per nucleus in control cells, 84 in ActD-treated cells, $P=0.07$ ) (Fig. 1B). Similarly, the fraction of GFP-TRF1 foci colocalizing with TERRA foci was similar in control and ActD-treated cells $(6.4 \%$ in control cells, $7.3 \%$ in ActDtreated cells, $P=0.16$ ) (Fig. 1B). We therefore conclude that ActD inhibited ongoing TERRA transcription without perturbing TERRA steady-state levels or telomeric localization.

We then monitored telomere movement by time-lapse microscopy. For image analysis, we chose cells in which the GFP-TRF1 signal was moderate and the fluorescent telomeric foci were clearly distinguishable from the diffuse GFP fluorescent background within the nucleus in order to avoid secondary effects exerted by heavily overexpressed TRF1 on telomere dynamics (data not shown). Moreover, to exclude from the analysis positional changes deriving from cell or nuclear movement, we measured the relative distance between two randomly chosen neighboring telomeres over the entire duration of the movie. In untreated cells, we measured a mean diffusion coefficient for telomeres of $5.0 \times$ $10^{-4} \mathrm{\mu m}^{2} / \mathrm{sec}$, a value comparable to the ones previously reported for telomeres in human osteosarcoma U2OS cells (diffusion coefficient: $5.8 \times 10^{-4} \mu^{2} / \mathrm{sec}$ ) or human bladder carcinoma UMUC3 cells $\left(2.315 \times 10^{-4}\right.$ to $15 \times$ $10^{-4} \mathrm{\mu m}^{2} / \mathrm{sec}$ ) (Molenaar et al. 2003; Wang et al. 2008). This indicates that GFP-TRF1 did not substantially alter telomere dynamics, at least in the chosen cells. We then compared the radius of confinement (ROC) and the step size (SS) of telomere movement, which reflect the constraint of chromatin movement and the change in distance covered by a telomere for a specific interval of time $(\Delta t)$, respectively (Qian et al. 1991; Marshall et al. 1997). In untreated cells telomeres traveled in a constrained random march with a ROC of $0.1 \mu \mathrm{m}$ for $\Delta \mathrm{t}$ of $7 \mathrm{sec}$ and a SS of $0.056 \mu \mathrm{m}$ for $\Delta \mathrm{t}$ of $1 \mathrm{sec}$ (Fig. 1C-E; Supplemental Fig. S2). In ActD-treated cells, telomeres were significantly less mobile and roamed smaller nuclear areas (ROC: $0.07 \mu \mathrm{m}$; SS: 0.050 $\mu \mathrm{m})$ (Fig. 1C-E; Supplemental Fig. S2). We obtained similar results with U2OS cells expressing GFP-TRF1 (Fig. 2). Thus, the ActD-dependent impairment of telomere dynamics is not cell line specific.
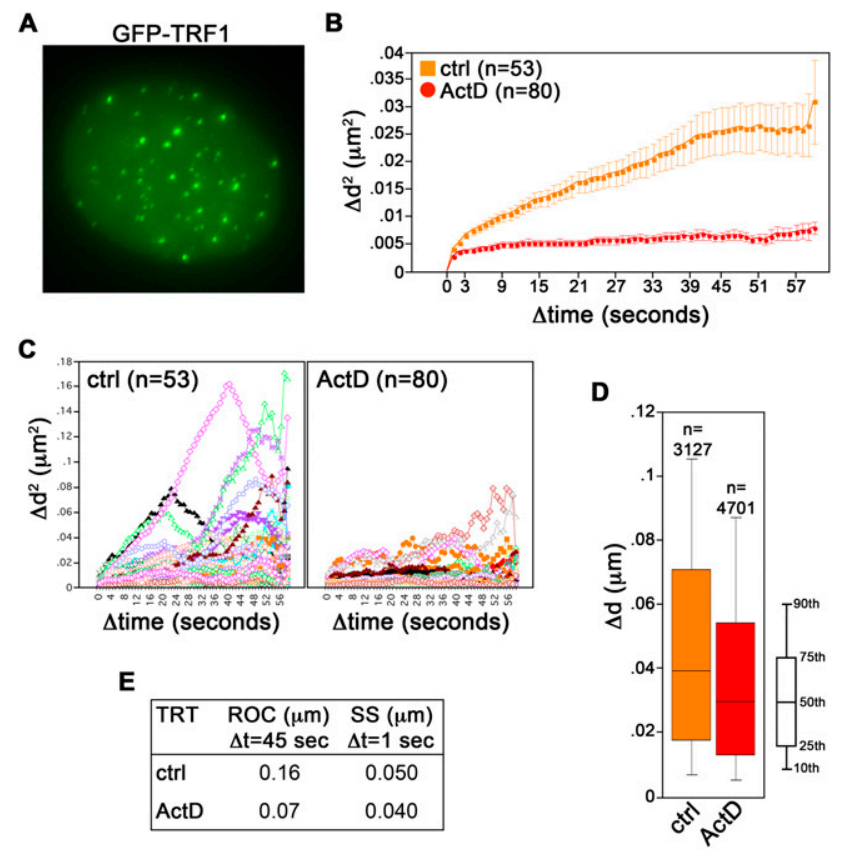

FIGURE 2. Transcription-dependent movement of natural telomeres in $\mathrm{U} 2 \mathrm{OS}$ cells. (A) U2OS cells were infected with retroviruses expressing GFP-TRF1 fusion protein (shown in green). (B-E) Live imaging of GFP-TRF1-tagged telomeres in cells treated with Actinomycin D (ActD) or left untreated (control cells: ctrl) was performed at a single focal plane every $1 \mathrm{sec}$ over $60 \mathrm{sec}$. Image analysis was as in Figure 1. (C) Single tracks of GFP-TRF1-tagged telomeres are shown. All $P$-values are $<0.0001$, and therefore are not indicated.

We then stimulated telomere transcription by heat shocking GFP-TRF1 cells at $42^{\circ} \mathrm{C}$ for $1 \mathrm{~h}$ in the presence or absence of ActD during the last $30 \mathrm{~min}$ of heat shock (HS). qRT-PCR experiments revealed augmented $15 \mathrm{q}$ and $\mathrm{Xp} / \mathrm{Yp}$ TERRA transcript levels both in heat-shocked cells and in heat-shocked cells treated with ActD (Supplemental Fig. S1C). Intriguingly, we did not observe a similar induction for 10q TERRA molecules (Supplemental Fig. S1C), suggesting that different chromosome ends might react to HS with different transcriptional kinetics. Heat-shocked nuclei contained brighter TERRA foci than untreated cells and the fraction of these foci colocalizing with GFP-TRF1 was significantly increased (13\% in HS-treated cells, $P<0.001$ vs. untreated cells) (Fig. 1B; Supplemental Fig. S1D). In cells treated with both HS and ActD, the average fraction of GFP-positive telomeres containing TERRA per nucleus was increased as compared with cells treated only with HS (19\%, $P<0.001$ vs. HS-treated cells) (Fig. 1B; Supplemental Fig. S1D). Altogether, these data are consistent with the notion that heat shock stimulates TERRA transcription (Schoeftner and Blasco 2008) and suggest that heat shock does not compromise the inherent ability of TERRA to localize at telomeres. In addition, it seems that transcription induction might contribute to the release of TERRA from telomeric heterochromatin in heat-shocked cells. Heat-shocked telomeres moved significantly faster than control telomeres 
(ROC: $0.15 \mu \mathrm{m}$; SS: $0.084 \mu \mathrm{m}$ ) (Fig. $1 \mathrm{C}-\mathrm{E}$ ), suggesting that transcription induction stimulates telomere motion. Consistently, ActD abolished this increased motility (ROC: $0.054 \mu \mathrm{m}$; SS: $0.034 \mu \mathrm{m}$ ) (Fig. 1C-E; Supplemental Fig. S2) in spite of the increased TERRA cellular levels and the increased localization of TERRA at telomeres. Altogether these observations reveal that telomere motion positively correlates with the transcriptional state of the cell and suggest that transcription per se, rather than TERRA association to telomeres, sustains telomere motion.

\section{DNA methyltransferases 1 and $3 b$ restrain telomere motion}

TERRA CpG-island promoters are completely demethylated in a HCT116-derived human cancer cell line deficient for DNA methyltransferase (DNMT) 1 and $3 \mathrm{~b}$ genes (double KO: DKO). As a consequence, RNAPII binding to TERRA promoters and TERRA steady-state levels are considerably increased (Nergadze et al. 2009). We infected parental (par) and DKO cells with GFP-TRF1 retroviruses (Fig. 3A), treated them with ActD, and analyzed telomere motion as above. In both cell lines, GFP-TRF1 was expressed at levels comparable to endogenous TRF1 (Supplemental Fig. S3A) and, consequently, telomere length was not perturbed in infected cells as compared with uninfected control cells (Supplemental Fig. S3B). As expected, the number and the intensity of TERRA foci was substantially increased in DKO cells as compared with par cells (Fig. 3B; Supplemental Fig. $\mathrm{S} 3 \mathrm{D})$. Alongside, the average fraction of GFP-TRF1-tagged telomeres colocalizing with TERRA foci was strikingly higher in DKO than in par cells (3\% in par cells, $21 \%$ in DKO cells, $P<0.001$ ) (Fig. 3B; Supplemental Fig. S3D). ActD did not significantly affect the cellular levels of TERRA transcripts produced from $10 \mathrm{q}$ and $15 \mathrm{q}$ (Supplemental Fig. S3C) nor the fraction of telomeres containing detectable TERRA in either cell line (3.5\% in ActD-treated par cells, $P=0.4$ vs. untreated par cells; $21 \%$ in ActD-treated DKO cells, $P=0.7$ vs. untreated DKO cells) (Fig. 3B; Supplemental Fig. S3D).

Live imaging of GFP-TRF1 foci revealed a diffusion coefficient of telomeres in par cells comparable to previously reported ones $\left(5.0 \times 10^{-4} \mathrm{\mu m}^{2} / \mathrm{sec}\right.$ ) (Molenaar et al. 2003; Wang et al. 2008), while it was substantially increased in DKO cells $\left(1.0 \times 10^{-3} \mu \mathrm{m}^{2} / \mathrm{sec}\right)$. Similarly, SS and ROC were augmented in DKO cells (SS: $0.066 \mu \mathrm{m}$ for $\Delta \mathrm{t}$ of $1 \mathrm{sec}$, ROC: $0.1 \mu \mathrm{m}$ for $\Delta \mathrm{t}$ of $7 \mathrm{sec}$ ) (Fig. $3 \mathrm{C}-\mathrm{E}$ ) versus par cells (SS: $0.058 \mu \mathrm{m}$, ROC: $0.09 \mu \mathrm{m}$ for $\Delta \mathrm{t}$ of $7 \mathrm{sec}$ ) (Fig. $3 \mathrm{C}-\mathrm{E}$ ). ActD dampened telomere motion in both cell lines (par ROC: $0.06 \mu \mathrm{m}$, par SS: $0.041 \mu \mathrm{m}$; DKO ROC: $0.08 \mu \mathrm{m}$, DKO SS: $0.056 \mu \mathrm{m}$ ) (Fig. 3C-E; Supplemental Fig. S2) although telomere movements remained less constrained in ActD-treated DKO cells than in ActD-treated par cells, possibly due to the fact that telomeres are overall shorter in DKO than in parental cells (Supplemental Fig. S3B). We
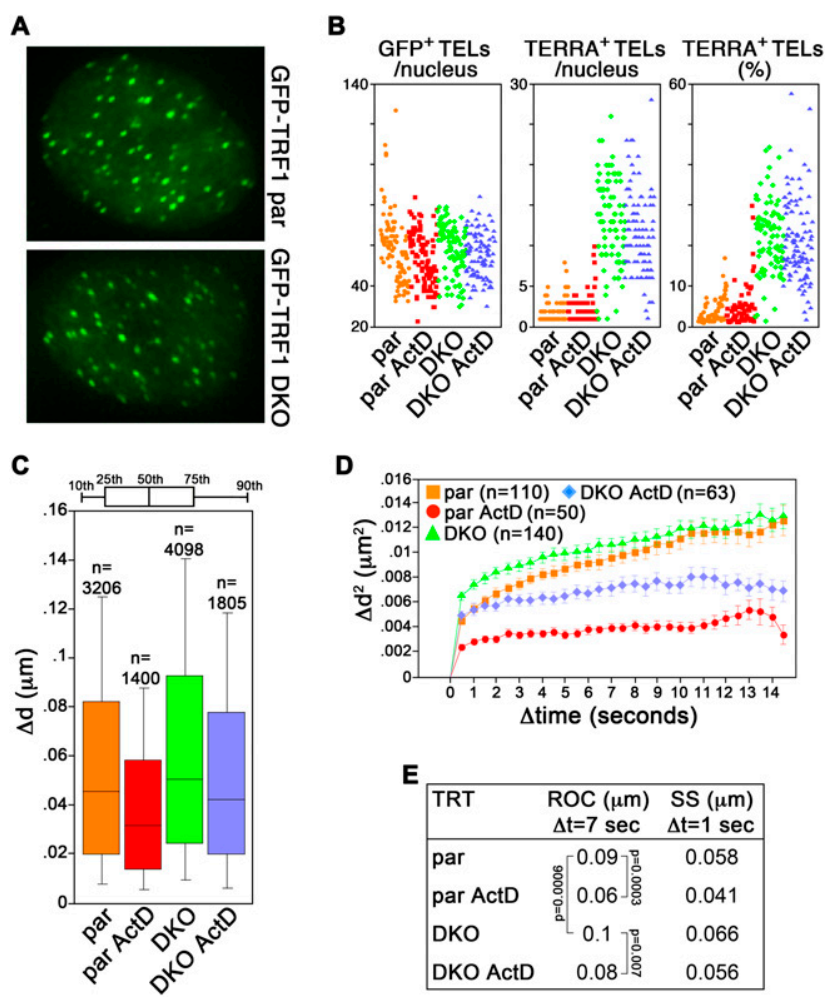

FIGURE 3. DNMT 1 and $3 \mathrm{~b}$ restrain telomere movements. $(A)$ Visualization of GFP-TRF1 in HTC116 parental (par) and DNMT1 and DNMT3b double knocked-out (DKO) cells. (B) Scatter plots showing the number of $\mathrm{GFP}^{+}$TELs (left), TERRA ${ }^{+}$TELs (middle), and percentage of TERRA ${ }^{+}$TELs (right) per nucleus from RNA FISH analysis of par and DKO cells expressing GFP-TRF1 treated as indicated. Each colored dot represents data from one nucleus. A total of $\sim 100$ nuclei from two independent experiments were analyzed per treatment. $(C-E)$ Cells were treated with ActD for $30 \mathrm{~min}$ before live imaging or left untreated. Live imaging and analysis were performed as in Figure 1. All $P$-values are $<0.0001$ unless indicated.

conclude that DNMT1 and DNMT3b constrain telomere motion, likely through $\mathrm{CpG}$ methylation-mediated inhibition of TERRA transcription. Moreover, as for HeLa cells treated with ActD or with heat shock, the recorded changes in telomere dynamics appear not to derive from TERRA mislocalization at telomeres.

\section{Transcription influences centromere dynamics in human cancer cells}

Transcription of satellite III centromeric sequences has been reported for a subset of human chromosomes and it is stimulated by heat shock (Rizzi et al. 2003). To test whether transcription influences the motility of centromeric heterochromatin, we made use of an established HeLa-derived cell line expressing the centromeric protein CENPA N-terminally fused with GFP (GFP-CENPA) (Jaqaman et al. 2010). The fusion protein colocalized with endogenous CENPI, confirming its centromeric localization (Fig. 4A). As for GFP-TRF1 cells, we treated GFP-CENPA cells with 
A

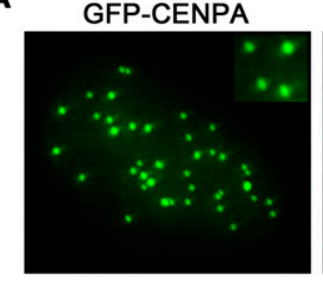

$\mathbf{B}$

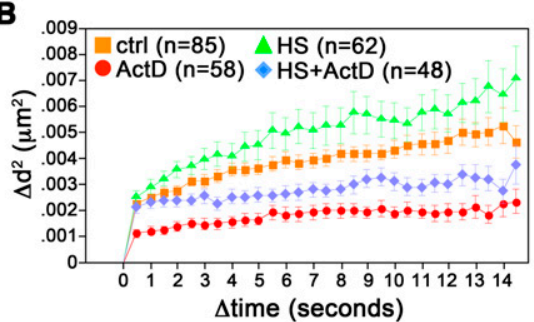

CENPI

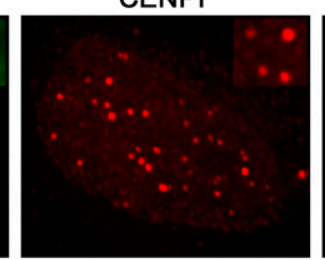

D
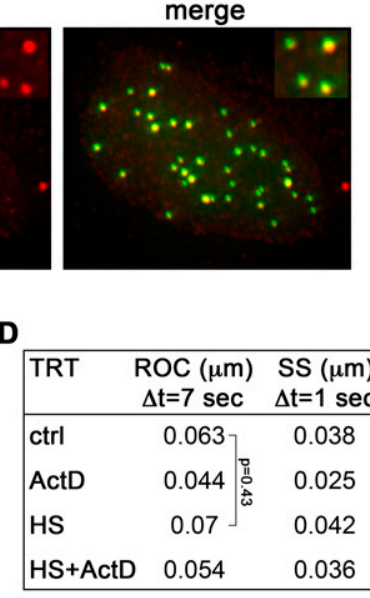

C

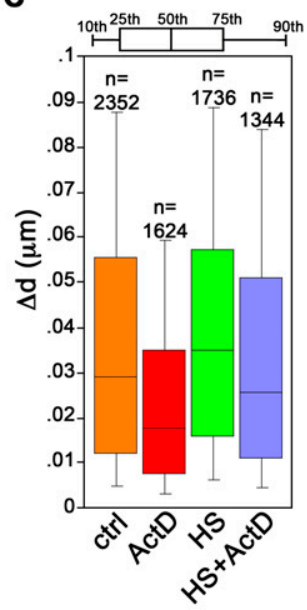

FIGURE 4. Centromere movement correlates with the transcriptional state of the cell. (A) HeLa cells expressing GFP-CENPA (green) were stained using antibodies against the endogenous centromeric protein CENPI (in red). ( $B-D$ ) Cell treatments and live imaging were performed as in Figure 1. All $P$-values are $<0.0001$ unless indicated.

ActD for $30 \mathrm{~min}$, either in the presence or absence of heat shock. Already in untreated samples, GFP-CENPA-tagged centromeres were significantly less mobile than GFP-TRF1tagged telomeres (ROC: $0.063 \mu \mathrm{m}$, SS: $0.038 \mu \mathrm{m}$ ) (Fig. 4B-D), revealing a more constrained motion associated with centromeric regions. As for telomeres, ActD treatment suddenly dampened centromere dynamics (ROC: $0.044 \mu \mathrm{m}$, SS: 0.025 $\mu \mathrm{m}$ ) (Fig. 4B-D). A statistically significant increase in SS $(0.042 \mu \mathrm{m})$, but not in ROC, was observed in heat-shocked cells. The movement enhancement induced by HS was abolished by concomitant treatments with ActD (ROC: $0.054 \mu \mathrm{m}$, SS: $0.036 \mu \mathrm{m}$ ) (Fig. 4B-D). Therefore, it appears that transcription regulates the movements not only of telomeres, but also of centromeres, and likely of heterochromatin at large.

\section{Transcription of a unique telomere increases its mobility}

The above-described approaches affect transcription of chromatin at large. To overcome this limitation we combined the telomere-seeding phenomenon with the inducible gene expression Tet-ON technology (Barnett et al. 1993; Gossen et al. 1995) and engineered a human cell line carrying "transcriptionally inducible telomeres" (tiTELs). We constructed a telomere seeding plasmid comprising a hygromycin resistance gene, an array of Lac operator (LacO) repeats (Robinett et al. 1996), a doxycycline (DOX)-inducible cytomegalovirus (CMV) promoter, and a (TTAGGG) $)_{n}$ telomeric array. The CMV promoter and the telomeric tract are separated by a $\sim 1.6-\mathrm{kb}-$ long unique sequence ("transcriptionally inducible subtelomere": tiSUBTEL) (Fig. 5A). We transfected linearized seeding plasmids into HeLa cells expressing the reverse Tet repressor and isolated stably transfected clonal populations. Telomere restriction fragment (TRF) analysis using tiSUBTEL probes as well as single telomere length analysis (STELA) (Baird et al. 2003) revealed that tiTEL seeding occurred in one clone (cl32) and that tiTEL length $(2.5-5 \mathrm{~kb})$ was comparable to the one of natural telomeres $(2-4.5 \mathrm{~kb})$ within the same cell line (Supplemental Fig. S5A,B). Further confirming the terminal location of tiTELs, dot-blot hybridization of genomic DNA digested with the BAL31 exonuclease disclosed a progressive disappearance of tiTEL sequences throughout the BAL31 digestion time course (Supplemental Fig. S5C). DNA FISH experiments performed using peptide nucleic acid (PNA) probes corresponding to LacO repeats revealed that out of 164 metaphases scored, 91\% carried two tiTELs, $8 \%$ one single tiTEL, and $1 \%$ had no tiTEL signal (Fig. 5B). We then stably transfected cl32 cells with plasmids expressing yellow fluorescence protein fused in frame with Lac repressor (LacI-YFP) (Tsukamoto et al. 2000). In interphase nuclei, tiTELs were detected as discrete fluorescent foci (Fig. 5C). qRT-PCR experiments revealed that DOX induced tiTEL transcription into transcriptionally inducible TERRA (tiTERRA) already within $3 \mathrm{~h}$, and prolonged (24-h) treatments induced a further accumulation of cellular tiTERRA (Fig. 5D). As expected, individual tiTERRA molecules comprised both telomeric 5'-UUAGGG-3' repeats and tiSUBTEL RNA sequences (Supplemental Fig. S4D). Given the identity of the two tiTELs within the same cell, our experimental approach does not allow us to distinguish between transcripts deriving from each of the two tiTELs.

For image analysis, the positional changes of a tagged tiTEL over time were computed after aligning the images of the same nucleus taken at each time point using the background fluorescence of the unbound LacI-YFP (Fig. 5C), 
A

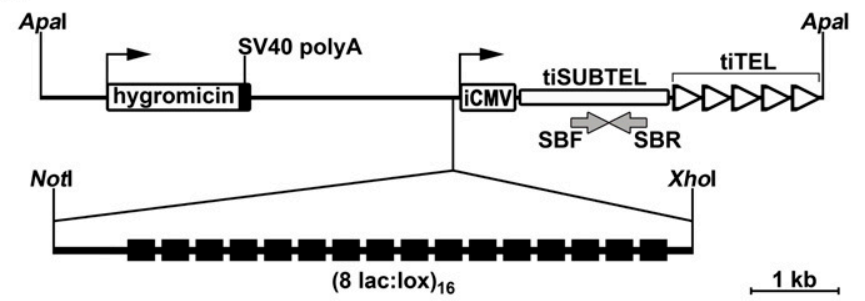

C

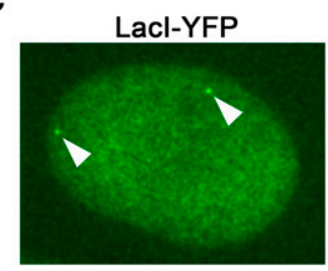

D

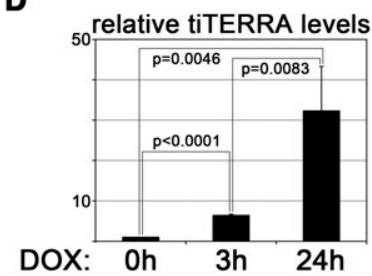

E

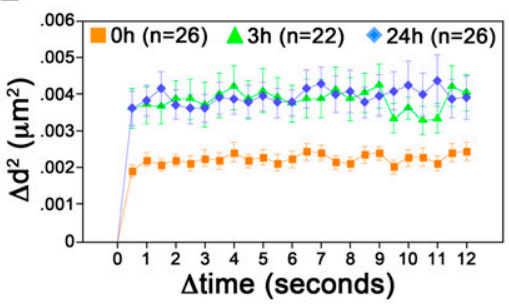

G

\begin{tabular}{|c|c|c|}
\hline $\operatorname{DOX}(\mathrm{h})$ & $\begin{array}{c}\mathrm{ROC}(\mu \mathrm{m}) \\
\Delta \mathrm{t}=7 \mathrm{sec}\end{array}$ & $\begin{array}{l}\text { SS }(\mu \mathrm{m}) \\
\Delta \mathrm{t}=1 \mathrm{sec}\end{array}$ \\
\hline 0 & \multirow{3}{*}{\multicolumn{2}{|c|}{ 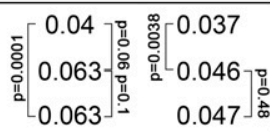 }} \\
\hline 3 & & \\
\hline 24 & & \\
\hline
\end{tabular}

B

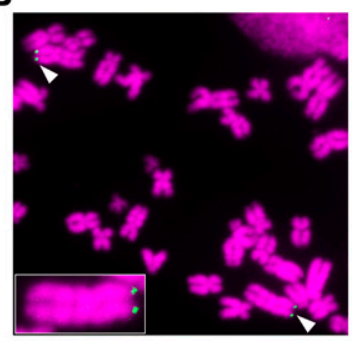

F

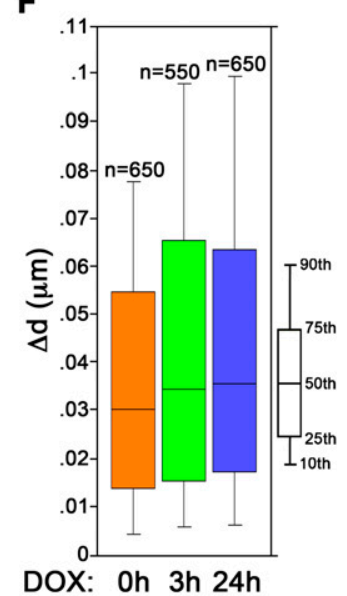

FIGURE 5. "Transcriptionally inducible telomeres" (tiTELs). (A) Sketch of the telomere seeding plasmid. (iCMV) Inducible CMV promoter; (SBF and SBR) oligonucleotides used in RT-PCR experiments; (black boxes) LacO repeats. (B) Partial cl32 metaphase hybridized in situ with fluorescently labeled LacO-repeat DNA oligonucleotides (green). DAPI-stained DNA is in magenta. White arrowheads point to tiTELs. The small tiTEL-containing chromosome on the right is enlarged in the inset to help visualization. $(C)$ Visualization of tiTELs as bright nuclear foci (white arrowheads) upon stable expression of LacI-YFP (green). The nuclear territory is marked by a diffuse signal. (D) Quantitative RT-PCR analysis of tiTERRA levels upon doxycycline (DOX) treatment. TiTERRA values are expressed as fold increase over untreated samples. Bars and error bars are averages and standard deviations from either two (3-h) or three (24-h) independent experiments. $P$-values are indicated. (E-G) For live imaging, images were taken at a single focal plane every $0.5 \mathrm{sec}$ over $15 \mathrm{sec}$. (E) Average mean square change in distance of tiTELs. The number of tracked tiTELs is indicated $(n)$. (F) Distribution of SS for $\Delta \mathrm{t}$ of $1 \mathrm{sec}$. The number of data points is indicated $(n)$. (G) Values for tiTEL ROC and SS calculated for the indicated DOX treatments. All $P$-values are $<0.001$ unless indicated.

thus excluding that the changes in tiTEL position derived from nuclear movements occurring during imaging. In untreated cells, tiTELs moved with a ROC of $0.04 \mu \mathrm{m}$ for $\Delta \mathrm{t}$ of $7 \mathrm{sec}$ and a SS of $0.037 \mu \mathrm{m}$ for $\Delta \mathrm{t}$ of $1 \mathrm{sec}$ (Fig. 5E-G; Supplemental Fig. S2). In samples treated with DOX for 3 or $24 \mathrm{~h}$, tiTELs displayed significantly increased dynamics (ROC: $0.063 \mu \mathrm{m}$ for both treatments; SS: 0.046 and 0.047 $\mu \mathrm{m}$ for DOX, $3 \mathrm{~h}$ and $24 \mathrm{~h}$, respectively) (Fig. $5 \mathrm{E}-\mathrm{G}$; Supplemental Fig. S2). DOX treatments of 3 and $24 \mathrm{~h}$ enhanced tiTEL motility to similar extents, while leading to different tiTERRA levels (Fig. 5D). Therefore, tiTEL transcription, rather than tiTERRA accumulation, fuels tiTEL motion. As for the qRT-PCR-based analysis of tiTERRA induction, we cannot discriminate between the two different tiTELs within the same cell during live imaging. Nevertheless, since imaged tiTELs were chosen randomly and no clear bimodal distribution of tiTEL movements was recorded (Supplemental Fig. S2), we conclude that the two tiTELs move with similar dynamics. Finally, DOX treatments did not affect telomere motion in HeLa GFP-TRF1 cells
(Supplemental Fig. S5), ruling out that the changes in tiTEL movements were due to secondary effects exerted by DOX.

\section{Transcription does not affect tiTEL stability}

To test whether induced transcription affected tiTEL length, we used STELA. In untreated cells tiTELs had an average length of $\sim 3 \mathrm{~kb}$, and no significant change was measured in cells treated with DOX for 3 or $24 \mathrm{~h}$ (Fig. 6A). On the contrary, prolonged cell culturing in the presence of the telomerase inhibitor BIBR1532 (Damm et al. 2001) led to progressive shortening of tiTELs as well as of natural $\mathrm{Xp} / \mathrm{Yp}$ telomeres (Supplemental Fig. S4B), confirming that tiTELs are maintained by telomerase, and validating our STELA analysis. To test whether transcription induction could hinder tiTEL integrity, we performed DNA-FISH experiments using combinations of PNA probes recognizing telomeric and $\mathrm{LacO}$ repeats. The fraction of $\mathrm{LacO}$-positive chromosomes remained similar both in DOX-treated and 
A

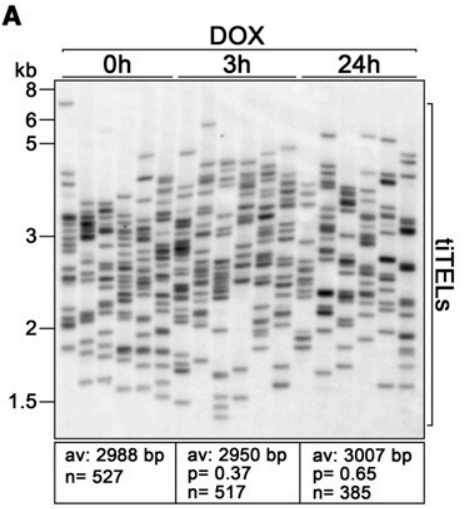

C

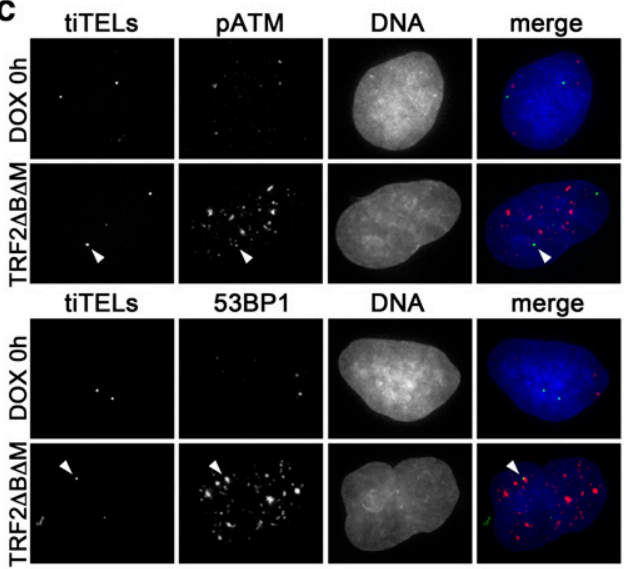

D

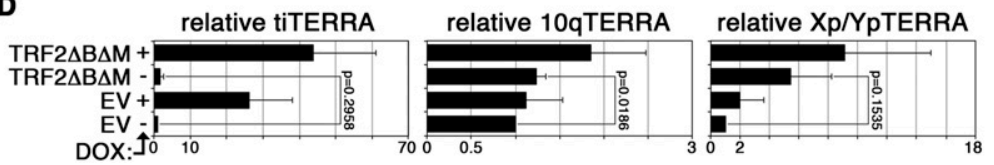

FIGURE 6. Transcription induction does not compromise tiTEL stability. (A) STELA analysis of tiTEL length in cells treated as indicated. Average (av) telomere length is indicated together with the number ( $\mathrm{n}$ ) of tiTELs analyzed. Molecular weights are on the left in kilobases. (B) DNA FISH on metaphase chromosomes prepared from cl32 cells treated as indicated. TiTELs were identified with LacO PNA probes (in green), telomeric sequences (telo) were detected using telomeric PNA probes (in red). DAPI-stained DNA is in blue. The white and black arrowheads point to the tiTEL enlarged at the top (three left panels) and to an example of telomere free end ${ }^{*}$ ) enlarged at the top (two right panels), respectively. Single channels and merged (mg) channels are shown. The fraction of telomere-free tiTELs (TFtiTELs), and endogenous telomere-free ends (TFendTELs) are indicated. Experiments were performed in duplicate and the cumulative number of analyzed tiTELs and endTELs are indicated (n) (C) cl32 cells expressing LacI-YFP were treated with DOX or infected with retroviruses expressing TRF2 $\Delta \mathrm{B} \Delta \mathrm{M}$. TiTELs were visualized using the YFP fluorescence (in green in the merge panels), while ATM phosphorylated at Serine 1981 (pATM) and 53BP1 were visualized by indirect immunofluorescence (in red). The arrowheads point to tiTELs colocalizing with pATM and with 53BP1. Bar graphs represent the fraction of tiTELs colocalizing with pATM or with 53BP1. Bars and error bars are averages and standard deviations from three independent experiments. (D) Quantitative RT-PCR analysis of tiTERRA and of endogenous TERRA transcribed from 10q and Xp/Yp chromosome ends in cl32 cells infected with TRF $2 \Delta \mathrm{B} \Delta \mathrm{M}$-expressing retroviruses or with empty vector (EV) retroviruses. TERRA values are expressed as fold increase over EV-infected, untreated cells. Bars and error bars are averages and standard deviations from either two (Xp/Yp) or three (tiTERRA and 10q) independent experiments; $P$-values are indicated.

untreated cells (data not shown). TiTELs devoid of detectable telomeric signals were never observed either in untreated or DOX-treated cells, while telomere-free endogenous chro- mosome ends (endTELs) could be occasionally detected (Fig. 6B). Finally, we tested the accumulation of the DNA damage factors ATM phosphorylated at Serine 1981 (pATM) and 53BP1 at LacI-YFPtagged tiTELs by indirect immunofluorescence. In untreated cells, $\sim 2 \%$ and $1 \%$ of tiTELs colocalized with pATM and 53BP1 foci, respectively. DOX treatments did not increase the fraction of tiTELs colocalizing with either marker (Fig. 6C). On the contrary, expression of a dominant-negative allele of TRF2 (TRF2 $\Delta \mathrm{B} \Delta \mathrm{M}$ ) increased significantly the fraction of tiTELs colocalizing with both markers, indicating that tiTELs can undergo uncapping, and validating our experimental approach (Fig. 6C). Although we cannot rule out that very mild or transient alterations of tiTEL length and integrity might have escaped our analysis, we conclude that transcription induction does not noticeably affect tiTEL stability, and the increased tiTEL dynamics observed upon DOX treatments are not a consequence of severe tiTEL shortening or uncapping.

It has been reported that human fibroblasts depleted for TRF2 accumulate TERRA molecules in a p53-dependent manner (Caslini et al. 2009). We therefore quantified the steady-state levels of tiTERRA as well as of TERRA from 10q and $\mathrm{Xp} / \mathrm{Yp}$ chromosome ends in $\mathrm{cl} 32$ cells infected with TRF $2 \Delta B \Delta M$, but could not observe any robust increase in TERRA transcripts as compared with empty vector control cells (Fig. 6D). This lack of TERRA up-regulation upon telomere uncapping is likely to be a consequence of the fact that HeLa cells possess a compromised p53 pathway (Matlashewski et al. 1986).

\section{CONCLUSIONS}

We have revealed here that transcription of a telomere directly regulates its motion without generating overt telomere instability. We suggest that transcriptionmediated telomere movements could influence the homeostasis of functional and/or dysfunctional telomeres, possibly by removing or thinning out telomere-associated factors that restrict chromatin motility. TERRA cellular levels are down-regulated 
during S-phase progression (Porro et al. 2010; Flynn et al. 2011), suggesting that decreased transcription of telomeres could favor their replication by immobilizing telomeric sequences. Moreover, TRF2-less uncapped telomeres in mouse embryonic fibroblasts failed to fuse with each other when prevented to gain their maximal mobility through depletion of ATM or 53BP1 (Dimitrova et al. 2008). It is conceivable that the increased telomere mobility induced by uncapping could derive, at least in part, from enhanced telomere transcription in cells with intact p53. In this light, telomere transcription could favor the DNA damage response arising at dysfunctional telomeres and their fusion. A thorough employment of our tiTEL system will contribute to shed light on these exciting suppositions.

\section{MATERIALS AND METHODS}

\section{Plasmid construction}

To generate the GFP-TRF1 retroviral plasmid, a PCR fragment coding for the entire ORF of the Green Fluorescence Protein (EGFP) was cloned in-frame with TRF1 into the PLPC-Flag-TRF1 plasmid (kind gift from Titia de Lange). To generate the tiTEL seeding plasmid, a 1628-bp-long fragment corresponding to the Luciferase ORF (tiSUBTEL) was inserted downstream from a strong human cytomegalovirus (CMV) immediate-early promoter, followed by two tetracycline operator sequences. The Luciferase sequence was placed in an inverted orientation to avoid synthesis of mRNA molecules. Successively, a $\sim 1.2$-kb-long (TTAGGG)n stretch was excised from the plasmid pCMVTelo (kind gift from Eric Gilson) and cloned immediately downstream from the Luciferase sequence. The plasmid was digested with NotI and XhoI and ligated to a $\sim 6.4-$ $\mathrm{kb}$ fragment containing $128 \mathrm{LacO}$ repeats excised from the plasmid pJRC49 (kind gift from Wendy Bickmore). The LacI-YFP plasmid was a kind gift from David Spector.

\section{Cell culture}

HeLa, T-REx-HeLa (Invitrogen), HCT116, DKO (kind gifts from Bert Vogelstein), and U2OS cells were cultured in high-glucose D-MEM supplemented with $10 \%$ tetracycline-free fetal calf serum and penicillin/streptomycin (Invitrogen). Where indicated, $1 \mathrm{mM}$ BIBR1532 was added to the culture medium. Plasmid transfections were performed using the Lipofectamine 2000 reagent (Invitrogen) according to the manufacturer's instructions. Retroviruses were produced in HEK 293T cells according to standard procedures. Twenty-four hours after transfection or infection, cells were trypsinized and plated in medium containing $200 \mu \mathrm{g} / \mathrm{mL}$ hygromycin or $1.5 \mu \mathrm{g} / \mathrm{mL}$ puromycin (Fluka). To verify GFP-TRF1 expression, Western blot analysis was performed according to standard procedures using antibodies raised against human TRF1 (ab10579, Abcam) and GFP (11814460001, Roche). Images were acquired using a FluorChem HD2 system (Alpha Innoctech). Metaphase chromosome spreads were prepared by incubating cells with $200 \mathrm{ng} / \mathrm{mL}$ Colcemid for $3 \mathrm{~h}$. Cells were harvested, incubated in $0.075 \mathrm{M} \mathrm{KCl}$ at $37^{\circ} \mathrm{C}$ for $8 \mathrm{~min}$, and fixed in methanol/acetic acid (3:1).

\section{DNA FISH, RNA FISH, and indirect immunofluorescence}

DNA and RNA FISH were performed essentially as previously described (Azzalin et al. 2007). For DNA FISH, metaphases were treated with $0.005 \%$ pepsin diluted in $10 \mathrm{mM}$ glycine $(\mathrm{pH} 2)$ for $5 \mathrm{~min}$ at $37^{\circ} \mathrm{C}$ prior to hybridization. Integrated $\mathrm{LacO}$ sequences were detected using FITC-OO-AATTGTTATCCGCTCACAATTC PNA probes (Bio-Synthesis, Inc.). Telomeric DNA was detected using Cy3-OO-(CCCTAA) ${ }_{5}$ PNA probes (Panagene). TERRA was detected with a mixture of 1-5-kb-long telomeric DNA fragments (TeloA probe) strand specifically labeled by random priming with Cy3-dCTP, dTTP, and dATP (Perkin Elmer). For indirect immunofluorescence, cells were cultured on coverslips in 6-well plates. Cells were first washed in $1 \times$ PBS, followed by a preextraction of soluble material in $0.5 \%$ Triton-X 100 for $7 \mathrm{~min}$, on ice. Cells were fixed in $3.7 \%$ paraformaldehyde for $10 \mathrm{~min}$ and successively blocked in 5\% BSA dissolved in 0.05\% Tween-20, $1 \times$ PBS for $30 \mathrm{~min}$ at RT. Cells were incubated with primary antibodies dissolved in blocking solution for $1 \mathrm{~h}$ at RT. The following antibodies were used: rabbit polyclonal anti hRap1 (A300-568A, Bethyl Laboratories), rabbit polyclonal anti CENPI (kind gift from Patrick Meraldi), mouse monoclonal anti ATM phosphorylated at Serine 1981 (4526S, Cell Signaling), and rabbit polyclonal anti 53BP1 (21083, Abcam). After extensive washes in $0.05 \%$ Tween-20, $1 \times$ PBS cells were incubated with secondary antibodies conjugated with Alexa 564 (Invitrogen) for $45 \mathrm{~min}$ at RT, and washed. Coverslips were mounted on slides with Vectashield (Vector Laboratories, Inc.). Z-stacks of $0.2 \mu \mathrm{m}$ were taken using the Deltavision system from Applied Precision. For tiTEL colocalization with pATM and 53BP1, experiments were performed in triplicates and 120-150 tiTels were evaluated in each experiment. Averages, standard deviations, and $P$-values (two-tailed Student's $t$-test) were calculated using the Microsoft Excel software.

\section{Telomere length and BAL31 digestion analysis}

Genomic DNA was prepared using the Wizard Genomic DNA Purification kit (Promega) according to the manufacturer's instructions. For terminal restriction fragment (TRF) analysis of bulk telomeres, $5 \mu \mathrm{g}$ of genomic DNA were digested with HinfI and RsaI restriction enzymes, and for tiTEL TRF analysis, $15 \mu \mathrm{g}$ of genomic DNA were digested with XhoI. Digested DNA was fractionated in $0.7 \%$ agarose gels. Gels were dried, denatured in $0.4 \mathrm{~N} \mathrm{NaOH}, 0.6 \mathrm{M} \mathrm{NaCl}$, and hybridized for $16 \mathrm{~h}$ at $50^{\circ}-64^{\circ} \mathrm{C}$ with radioactively labeled TeloA or tiSUBTEL probes. Post hybridization washes were at $50^{\circ}-64^{\circ} \mathrm{C}$ in $1-0.2 \times$ SSC, $0.5 \%$ SDS. Radioactive signals were detected using a Typhoon FLA 9000 instrument (GE Healthcare). For STELA, $2 \mu \mathrm{g}$ of genomic DNA was digested with XhoI for $16 \mathrm{~h}$ and purified with the GeneClean Turbo Kit (MP Biomedicals). A total of $20 \mathrm{ng}$ of digested DNA was mixed with $1 \mu \mathrm{L}$ of $10 \mu \mathrm{M}$ telorette 3 oligonucleotide (5' $-\mathrm{TGC}$ TCCGTGCATCTGGCATCCCTAACC- $3^{\prime}$ ), incubated at $60^{\circ} \mathrm{C}$ for $10 \mathrm{~min}$, and cooled down at RT before adding $1 \mathrm{mM}$ ATP and $1 \mathrm{U}$ of T4 DNA ligase (New England Biolabs) in $10-\mu \mathrm{L}$ reactions. Ligations were performed at $35^{\circ} \mathrm{C}$ for $12 \mathrm{~h}$, followed by heat inactivation. A total of $400 \mathrm{pg}$ of ligated DNA was PCR amplified in $15-\mu \mathrm{L}$ reactions containing $75 \mathrm{mM}$ Tris- $\mathrm{HCl}(\mathrm{pH} 8.8), 200 \mathrm{mM}$ $\left(\mathrm{NH}_{4}\right)_{2} \mathrm{SO}_{4}, 0.01 \%$ Tween-20, $0.5 \mu \mathrm{M}$ Teltail oligonucleotide (5' TGCTCCGTGCATCTGGCATC-3' $\left.{ }^{\prime}\right), \quad 0.5 \mu \mathrm{M}$ tiTEL or $\mathrm{Xp} / \mathrm{Yp}$ 
sutelomere oligonucleotide (tiTEL: 5'-ACCGGGAGGTAGATG AGATG-3'; Xp/Yp: 5'-GTTGTCTCAGGGTCCTAGTG-3'), $0.3 \mu \mathrm{M}$ dNTPs, $1.5 \mu \mathrm{M} \mathrm{MgCl}{ }_{2}, 1.5 \mathrm{U}$ of Taq Thermoprime Polymerase (ABgene) and $0.15 \mathrm{U}$ of Pwo polymerase (Roche). PCR conditions were: $15 \mathrm{sec}$ at $94^{\circ} \mathrm{C}, 30 \mathrm{sec}$ at $65^{\circ} \mathrm{C}$, and $10 \mathrm{~min}$ at $68^{\circ} \mathrm{C}$ for 25 cycles. Amplification products were electrophoresed in $0.8 \%$ agarose gels, denatured, and hybridized in gel using radiolabeled tiSUBTEL or Xp/Yp subtelomere probes. Hybridizations were performed at $60^{\circ} \mathrm{C}$ for $16 \mathrm{~h}$, and post-hybridization washes were at $60^{\circ} \mathrm{C}$ in $0.2 \times$ SSC, $0.2 \%$ SDS. The length of single telomeres was calculated using the software Quantity One. In each lane the band with the lowest intensity was considered to represent 1 telomere. The number of telomeres represented by each band in every lane was computed based on the fold increase in intensity over this value. Averages and $P$-values (two-tailed Student's $t$-test) were calculated using the Statview (4.5) software. For BAL31 experiments, $20 \mu \mathrm{g}$ of undigested genomic DNA were incubated with $6 \mathrm{U}$ of BAL31 (New England Biolabs) over a time course of $3 \mathrm{~h}$. At the indicated time points, $2-\mu \mathrm{g}$ aliquots were taken and BAL31 was inactivated at $65^{\circ} \mathrm{C}$ for $15 \mathrm{~min}$ in the presence of 20 mM EGTA, followed by phenol/chloroform extraction. DNA was precipitated, dot blotted onto nylon membranes, denatured, and hybridized successively using radioactively labeled tiSUBTEL probes, TeloA probes, and Alu repeat oligonucleotide probes (5'-GTGATCCGCCCGCCTCGGCCTCCCAAAGTG-3').

\section{RT-PCR}

Total RNA was prepared using the NucleoSpin RNA II system (Macherey-Nagel) and subjected to three successive DNaseI (Qiagen) digestions. A total of 1-5 $\mu$ g of total RNA was reversetranscribed using the SUPERSCRIPT III Reverse transcriptase (GIBCO BRL), according to the manufacturer's instructions. Reverse transcription was performed using random hexamers or telomeric (CCCTAA $)_{5}$ oligonucleotides. cDNA was PCR amplified using the LightCycler 480 SYBR Green I Master mix (Roche) and a Rotor-Gene Q instrument (Qiagen). PCR oligonucleotides were SBF (5'-AGAATCTCACGCAGGCAGTT-3') and SBR (5'-CCAGGGAT TTCAGTCGATGT-3') for tiTERRA; c-mycF (5'-CCTACCTC TTCAACGACAGC-3') and c-mycR (5'-CTCTGACCTTTTGCCAG GAG-3') for c-myc; 10qF (5'-GAATCCTGCGCACCGAGAT-3') and $10 \mathrm{qR}\left(5^{\prime}\right.$-CTGCACTTGAACCCTGCAATAC-3') for $10 \mathrm{q} ; 15 \mathrm{qF}$ $\left(5^{\prime}\right.$-CAGCGAGATTCTCCCAAGCTAAG-3') and 15qR (5'-AAC CCTAACCACATGAGCAACG- $\left.3^{\prime}\right)$ for $15 \mathrm{q} ; \mathrm{Xp} / \mathrm{YpF}$ (5'-GCAAAGA GTGAAAGAACGAAGCTT- $\left.3^{\prime}\right)$ and Xp/YpR (5'-CCCTCTGAAA GTGGACCAATCA-3') for Xp/Yp; U6F (5'-GGAATCTAGAACAT ATACTAAAATTGGAAC-3') and U6R (5'-GGAACTCGAGTTTG CGTGTCATCCTTGCGC-3' ${ }^{\prime}$ ) for U6 (normalization control). PCR cycles were as follows: $10 \mathrm{sec}$ at $98^{\circ} \mathrm{C}, 30 \mathrm{sec}$ at $60^{\circ} \mathrm{C}$ for 40 cycles. Averages, standard deviations, and $P$-values (two-tailed Student's $t$-test) were calculated using the Microsoft Excel software.

\section{Live cell imaging and image analysis}

The Deltavision Multiplex system (Applied Precision) was used for live fluorescence imaging of cells plated $24 \mathrm{~h}$ before imaging. Cells were imaged in Leibovitz medium devoid of phenol red supplemented with $30 \%$ fetal bovine serum (Invitrogen). Where indicated, cells were treated with $1 \mu \mathrm{g} / \mathrm{mL}$ doxycycline or $5 \mu \mathrm{g} / \mathrm{mL}$ Actinomycin D (Sigma). For heat shock, cells were incubated at $42^{\circ} \mathrm{C}$ for $1 \mathrm{~h}$. All heat-shocked samples were imaged at $42^{\circ} \mathrm{C}$, while all other samples were imaged at $37^{\circ} \mathrm{C}$. For image analysis, pairs of neighboring telomeres in the GFP-TRF1 cell lines were chosen randomly in independent nuclei, and the relative distance between the two tagged telomeres was measured for all time points using a script developed in MATLAB (Thakar et al. 2006). For image analysis of tiTELs, nuclei were aligned through their center of mass using the object stabilizer tool in the Huygen's Essential software. Images were deconvolved using the deconvolution tool for wide field microscopy in the Huygen's Essential software. TiTEL movement was tracked for all time points using a script developed in MATLAB (Thakar et al. 2006). The mean square change in distance $\left\langle\Delta \mathrm{d}^{2}\right\rangle$ was calculated for all possible values of $\Delta \mathrm{t}$ using a macro developed in the Microsoft Excel software (Thakar et al. 2006). The average value of $\left\langle\Delta \mathrm{d}^{2}\right\rangle$ was plotted using the StatView (4.5) software. Error bars in all of the graphs correspond to one standard error. ROC was calculated as the square root of the height of the graph for $\Delta t$ of $7 \mathrm{sec}$. SS was calculated for $\Delta \mathrm{t}$ of $1 \mathrm{sec}$ and the distribution was depicted as a box plot, where each horizontal bar represents a percentile. The horizontal bar in the middle of the box plot corresponds to the median (50th percentile) of the distribution. The diffusion coefficient (D) was calculated from the slope of the mean square change in distance, based on the equation $\left\langle\Delta \mathrm{d}^{2}\right\rangle=4 \mathrm{D}\langle\Delta \mathrm{t}\rangle$ (Qian et al. 1991) using the values of $\left\langle\Delta \mathrm{d}^{2}\right\rangle$ at $\Delta \mathrm{t}$ of 0.5 and 1.0 sec. Student's $t$-test and Mann-Whitney test were carried out using the StatView (4.5) software.

\section{SUPPLEMENTAL MATERIAL}

Supplemental material is available for this article.

\section{ACKNOWLEDGMENTS}

We thank J. Lingner, P. Reichenbach, P. Meraldi, W. Bickmore, T. de Lange, E. Gilson, B. Vogelstein, and D. Spector for reagents and help. We thank the ETHZ Light Microscopy Centre for microscope services and the members of our laboratory for inspiring discussions. This work was funded by the European Research Council (BFTERRA) and the Swiss National Science Foundation (3100A0-120090 and PP00P3-123356). C.M.B. received a fellowship from Boehringer Ingelheim Fonds.

Received July 30, 2011; accepted January 4, 2012.

\section{REFERENCES}

Arora R, Brun CM, Azzalin CM. 2010. TERRA: Long noncoding RNA at eukaryotic telomeres. Prog Mol Subcell Biol 51: 65-94.

Azzalin CM, Reichenbach P, Khoriauli L, Giulotto E, Lingner J. 2007. Telomeric repeat containing RNA and RNA surveillance factors at mammalian chromosome ends. Science 318: 798-801.

Baird DM, Rowson J, Wynford-Thomas D, Kipling D. 2003. Extensive allelic variation and ultrashort telomeres in senescent human cells. Nat Genet 33: 203-207.

Barnett MA, Buckle VJ, Evans EP, Porter AC, Rout D, Smith AG, Brown WR. 1993. Telomere directed fragmentation of mammalian chromosomes. Nucleic Acids Res 21: 27-36.

Boyle S, Gilchrist S, Bridger JM, Mahy NL, Ellis JA, Bickmore WA. 2001. The spatial organization of human chromosomes within the nuclei of normal and emerin-mutant cells. Hum Mol Genet 10: 211-219. 
Caslini C, Connelly JA, Serna A, Broccoli D, Hess JL. 2009. MLL associates with telomeres and regulates telomeric repeat-containing RNA transcription. Mol Cell Biol 29: 4519-4526.

Chuang $\mathrm{CH}$, Carpenter AE, Fuchsova B, Johnson T, de Lanerolle P, Belmont AS. 2006. Long-range directional movement of an interphase chromosome site. Curr Biol 16: 825-831.

Chubb JR, Boyle S, Perry P, Bickmore WA. 2002. Chromatin motion is constrained by association with nuclear compartments in human cells. Curr Biol 12: 439-445.

Damm K, Hemmann U, Garin-Chesa P, Hauel N, Kauffmann I, Priepke H, Niestroj C, Daiber C, Enenkel B, Guilliard B, et al. 2001. A highly selective telomerase inhibitor limiting human cancer cell proliferation. EMBO J 20: 6958-6968.

Deng Z, Norseen J, Wiedmer A, Riethman H, Lieberman PM. 2009. TERRA RNA binding to TRF2 facilitates heterochromatin formation and ORC recruitment at telomeres. Mol Cell 35: 403-413.

Dimitrova N, Chen YC, Spector DL, de Lange T. 2008. 53BP1 promotes non-homologous end joining of telomeres by increasing chromatin mobility. Nature 456: 524-528.

Flynn RL, Centore RC, O’Sullivan RJ, Rai R, Tse A, Songyang Z, Chang S, Karlseder J, Zou L. 2011. TERRA and hnRNPA1 orchestrate an RPA-to-POT1 switch on telomeric single-stranded DNA. Nature 471: 532-536.

Gossen M, Freundlieb S, Bender G, Muller G, Hillen W, Bujard H. 1995. Transcriptional activation by tetracyclines in mammalian cells. Science 268: 1766-1769.

Hübner MR, Spector DL. 2010. Chromatin dynamics. Annu Rev Biophys 39: 471-489.

Jaqaman K, King EM, Amaro AC, Winter JR, Dorn JF, Elliott HL, McHedlishvili N, McClelland SE, Porter IM, Posch M, et al. 2010. Kinetochore alignment within the metaphase plate is regulated by centromere stiffness and microtubule depolymerases. J Cell Biol 188: $665-679$.

Jegou T, Chung I, Heuvelman G, Wachsmuth M, Gorisch SM, GreulichBode KM, Boukamp P, Lichter P, Rippe K. 2009. Dynamics of telomeres and promyelocytic leukemia nuclear bodies in a telomerase-negative human cell line. Mol Biol Cell 20: 2070-2082.

Luke B, Panza A, Redon S, Iglesias N, Li Z, Lingner J. 2008. The Ratlp $5^{\prime}$ to $3^{\prime}$ exonuclease degrades telomeric repeat-containing RNA and promotes telomere elongation in Saccharomyces cerevisiae. Mol Cell 32: 465-477.

Marshall WF, Straight A, Marko JF, Swedlow J, Dernburg A, Belmont A, Murray AW, Agard DA, Sedat JW. 1997. Interphase chromosomes undergo constrained diffusional motion in living cells. Curr Biol 7: 930-939.

Matlashewski G, Banks L, Pim D, Crawford L. 1986. Analysis of human p53 proteins and mRNA levels in normal and transformed cells. Eur J Biochem 154: 665-667.

Molenaar C, Wiesmeijer K, Verwoerd NP, Khazen S, Eils R, Tanke HJ, Dirks RW. 2003. Visualizing telomere dynamics in living mammalian cells using PNA probes. EMBO J 22: 6631-6641.
Nergadze SG, Farnung BO, Wischnewski H, Khoriauli L, Vitelli V, Chawla R, Giulotto E, Azzalin CM. 2009. CpG-island promoters drive transcription of human telomeres. RNA 15: 2186-2194.

Porro A, Feuerhahn S, Reichenbach P, Lingner J. 2010. Molecular dissection of telomeric repeat-containing RNA biogenesis unveils the presence of distinct and multiple regulatory pathways. Mol Cell Biol 30: 4808-4817.

Qian H, Sheetz MP, Elson EL. 1991. Single particle tracking. Analysis of diffusion and flow in two-dimensional systems. Biophys J 60: 910-921.

Rajapakse I, Groudine M. 2011. On emerging nuclear order. J Cell Biol 192: $711-721$.

Redon S, Reichenbach P, Lingner J. 2010. The non-coding RNA TERRA is a natural ligand and direct inhibitor of human telomerase. Nucleic Acids Res 38: 5797-5806.

Rizzi N, Denegri M, Chiodi I, Corioni M, Valgardsdottir R, Cobianchi F, Riva S, Biamonti G. 2003. Transcriptional activation of a constitutive heterochromatic domain of the human genome in response to heat shock. Mol Biol Cell 15: 543-551.

Robinett CC, Straight A, Li G, Willhelm C, Sudlow G, Murray A, Belmont AS. 1996. In vivo localization of DNA sequences and visualization of large-scale chromatin organization using lac operator/repressor recognition. J Cell Biol 135: 1685-1700.

Schoeftner S, Blasco MA. 2008. Developmentally regulated transcription of mammalian telomeres by DNA-dependent RNA polymerase II. Nat Cell Biol 10: 228-236.

Solovei I, Gaginskaya ER, Macgregor HC. 1994. The arrangement and transcription of telomere DNA sequences at the ends of lampbrush chromosomes of birds. Chromosome Res 2: 460-470.

Stixova L, Bartova E, Matula P, Danek O, Legartova S, Kozubek S. 2011. Heterogeneity in the kinetics of nuclear proteins and trajectories of substructures associated with heterochromatin. Epigenetics Chromatin 4: 5. doi: 10.1186/1756-8935-4-5.

Thakar R, Gordon G, Csink AK. 2006. Dynamics and anchoring of heterochromatic loci during development. J Cell Sci 119: 41654175.

Tsukamoto T, Hashiguchi N, Janicki SM, Tumbar T, Belmont AS, Spector DL. 2000. Visualization of gene activity in living cells. Nat Cell Biol 2: 871-878.

van Steensel B, de Lange T. 1997. Control of telomere length by the human telomeric protein TRF1. Nature 385: 740-743.

Vrbsky J, Akimcheva S, Watson JM, Turner TL, Daxinger L, Vyskot B, Aufsatz W, Riha K. 2010. siRNA-mediated methylation of Arabidopsis telomeres. PLoS Genet 6: e1000986. doi: 10.1371/journal. pgen.1000986.

Wang X, Kam Z, Carlton PM, Xu L, Sedat JW, Blackburn EH. 2008. Rapid telomere motions in live human cells analyzed by highly time-resolved microscopy. Epigenetics Chromatin 1: 4. doi: 10.1186/1756-8935-1-4.

Zhao R, Bodnar MS, Spector DL. 2009. Nuclear neighborhoods and gene expression. Curr Opin Genet Dev 19: 172-179. 

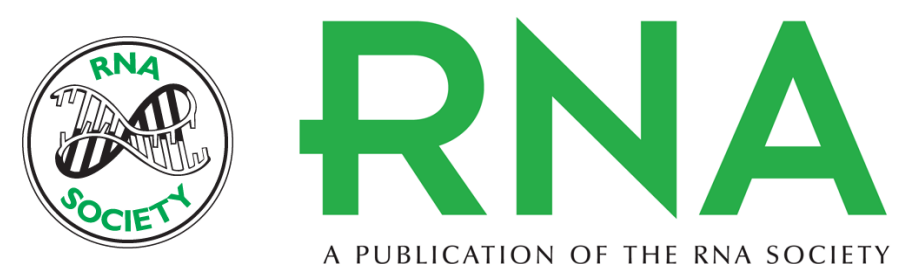

A PUBLICATION OF THE RNA SOCIETY

\section{Transcription regulates telomere dynamics in human cancer cells}

Rajika Arora, Catherine M. Brun and Claus M. Azzalin

RNA 2012 18: 684-693 originally published online February 22, 2012

Access the most recent version at doi:10.1261/rna.029587.111

Supplemental

Material

References

\section{License}

Email Alerting

Service
http://rnajournal.cshlp.org/content/suppl/2012/02/02/rna.029587.111.DC1

This article cites 36 articles, 11 of which can be accessed free at: http://rnajournal.cshlp.org/content/18/4/684.full.html\#ref-list-1 